\title{
Pour ou contre la ventilation à percussions intrapulmonaires en réanimation?
}

\section{Intrapulmonary percussive ventilation in the intensive care unit: a pro-con debate}

\author{
G. Riffard $\cdot$ M. Toussaint \\ Reçu le 31 août 2011 ; accepté le 5 décembre 2011 \\ C SRLF et Springer-Verlag France 2011
}

Résumé L'utilité de la ventilation à percussions intrapulmonaires (IPV) en réanimation fait l'objet de controverses en raison du manque de preuves scientifiques quant à ses principes d'action et à ses bénéfices thérapeutiques. L'impact de l'IPV rapporté dans la littérature médicale concerne l'amélioration du drainage bronchique, le recrutement de territoires pulmonaires mal ventilés et l'amélioration des échanges gazeux, particulièrement chez des patients manquant de participation active à d'autres techniques de kinésithérapie de désencombrement, du fait de la sédation, du manque de compliance, de l'instabilité clinique, de la faiblesse ou de la fatigue musculaire respiratoire. Le rôle préventif de l'IPV chez des patients souffrant de bronchopneumopathie chronique obstructive à risque élevé d'exacerbation est relativement bien décrit. L'emploi régulier de l'IPV chez les patients intubés ou trachéotomisés semble aider à limiter le taux de pneumonies nosocomiales et à réduire les durées de séjour en réanimation. Cependant, le manque de données scientifiques, la complexité de sa mise en œuvre et le manque de monitorage adéquat constituent un frein à son utilisation systématique. L'IPV pourrait trouver sa place en réanimation en cas d'échec des techniques conventionnelles de kinésithérapie respiratoire, mais son utilité mérite d'être mieux étudiée.

Mots clés Désencombrement · Kinésithérapie respiratoire · Ventilation à percussion intrapulmonaire $\cdot$ Réanimation

Abstract Effectiveness of intrapulmonary percussion ventilation (IPV) in the intensive care unit (ICU) remains

\section{G. Riffard $(\bowtie)$}

Service de rééducation fonctionnelle, CHU-hôpital Nord, CHU de Saint-Étienne, F-42055 Saint-Étienne cedex 02, France e-mail : guillaume.riffard@orange.fr

M. Toussaint

Ziekenhuis Inkendaal, centre de ventilation mécanique pour le domicile et centre neuromusculaire, Vlezenbeek, Belgique controversial due to the lack of evidence regarding its principles and therapeutic benefits. IPV impact in medical literature targets improvement of airway clearance and gas exchange as well as recruitment of poorly ventilated lung areas, particularly in patients lacking active participation in other techniques of chest physiotherapy because of sedation, clinical instability, respiratory muscle fatigue or weakness, or due to non-compliance. IPV preventive role in patients with chronic obstructive pulmonary disease at high risk of exacerbation is relatively well described. Regular IPV use in patients with tracheal intubation or tracheostomy seems to reduce the rate of hospital-acquired pneumonia and the length of ICU stay. However, lack of bibliographic support and complexity of implementation and adequate monitoring represent a significant barrier to its routine use in the ICU. IPV seems to find a place when conventional techniques of chest physiotherapy fail.

Keywords Airway clearance $\cdot$ Chest physiotherapy $\cdot$ Intrapulmonary percussive ventilation $\cdot$ Intensive care unit

\section{Introduction}

Dans les années 1980, les travaux du Dr F.M. Bird sur la ventilation à haute fréquence aboutirent à la mise au point de deux types très différents d'appareils : ceux délivrant la ventilation par percussions à haute fréquence ou high frequency percussive ventilation (HFPV) et ceux délivrant la ventilation à percussions intrapulmonaires ou intrapulmonary percussive ventilation (IPV).

La première est une technique particulière de ventilation artificielle, qui superpose aux cycles ventilatoires classiques délivrés à basse fréquence (10 à 30 cycles/min) des cycles à très faibles volumes délivrés à très haute fréquence (> 300 cycles/min) (Fig. 1). Elle a été proposée dans la ventilation du syndrome de détresse respiratoire aiguë [1]. Elle ne fait pas l'objet de cet article. 


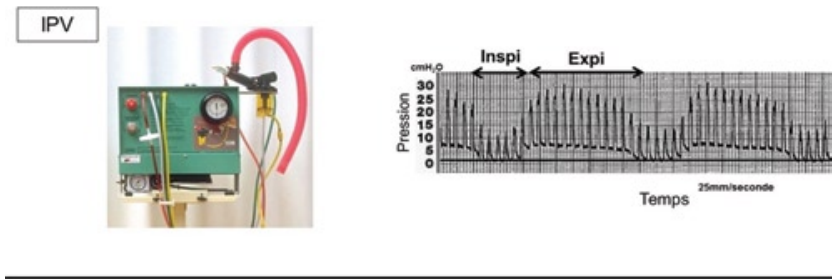

HFPV
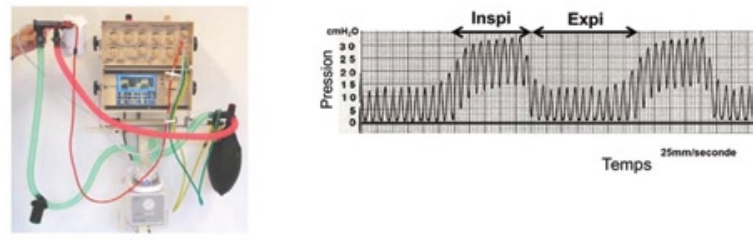

Fig. 1. Différence entre la ventilation à percussion intrapulmonaire (IPV) et la ventilation percussive à haute fréquence (HFPV) L'IPV, utilisée transitoirement lors des séances de kinésithérapie respiratoire, se superpose à la ventilation spontanée du patient. Noter la diminution de la pression lors de la phase inspiratoire (Inspi) et son augmentation lors de la phase expiratoire (Expi). L'HFPV est un mode de ventilation artificielle utilisé en continu. Comme en ventilation conventionnelle, les cycles inspiratoires correspondent à des cycles de montée en pression.

La seconde ou IPV est une technique instrumentale de kinésithérapie respiratoire utilisée pour améliorer la clairance des sécrétions bronchiques, et aussi les échanges gazeux. Elle consiste à administrer dans les voies aériennes de très faibles volumes courants, généralement inférieurs à l'espace mort physiologique, à haute fréquence (entre 60 et 600 cycles/min), tout au long du cycle respiratoire (Fig. 1).

L'IPV a été proposée dans la prise en charge respiratoire au long cours des maladies neuromusculaires [2], de la mucoviscidose [3,4] ou des bronchopneumopathies chroniques obstructives (BPCO) $[5,6]$. Seule son utilisation en réanimation sera abordée ici. Ne seront pas traitées les indications spécifiques au petit enfant, telles que les retards de résorption méconiale [7] et autres atteintes respiratoires des prématurés [8], la prise en charge d'atélectasies du petit enfant $[9,10]$ et la bronchiolite du nourrisson [11].

\section{Principes d'action de l'IPV}

L'IPV peut s'appliquer aux voies respiratoires en ventilation spontanée, via une sonde d'intubation ou une pièce buccale (Fig. 2). Elle peut également être utilisée pendant la ventilation mécanique conventionnelle sur intubation trachéale ou trachéotomie, au moyen d'un adaptateur spécifique (Fig. 3).

Le principe de son action est commun à toute ventilation à haute fréquence [12] : la succession des percussions crée au centre de la lumière bronchique un front de gaz qui progresse vers la périphérie du poumon, tandis qu'un contre-débit rétrograde s'établit de la périphérie vers la bouche, le long des

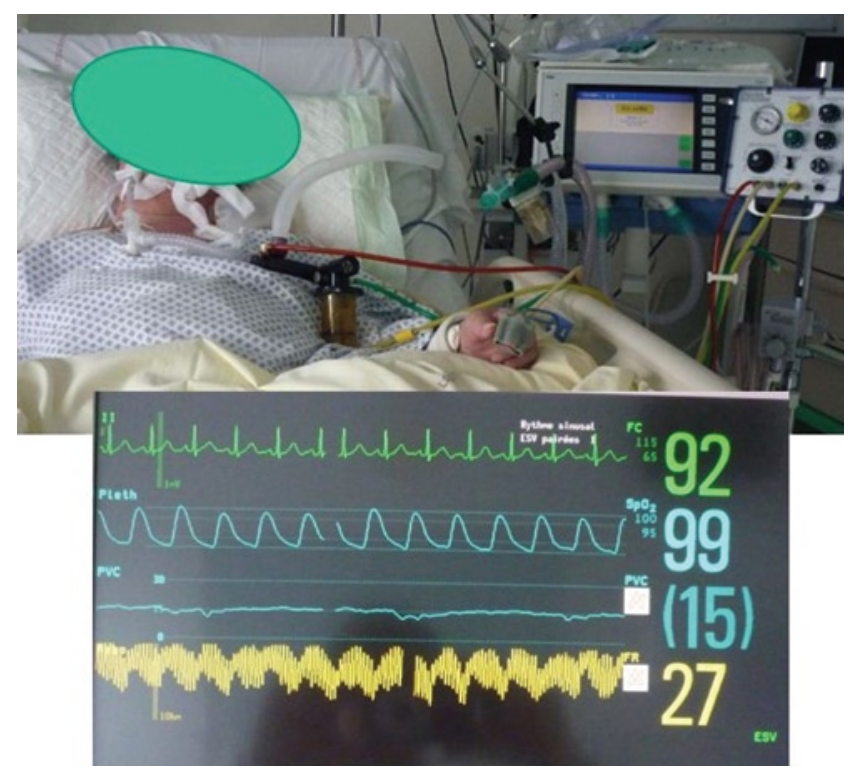

Fig. 2. Utilisation invasive de la ventilation à percussions intrapulmonaires (IPV)

Le patient est déconnecté du ventilateur conventionnel, puis relié à l'appareil délivrant l'IPV. Noter sur le moniteur du patient, l'apparition des percussions qui se superposent à la courbe de fréquence respiratoire spontanée.

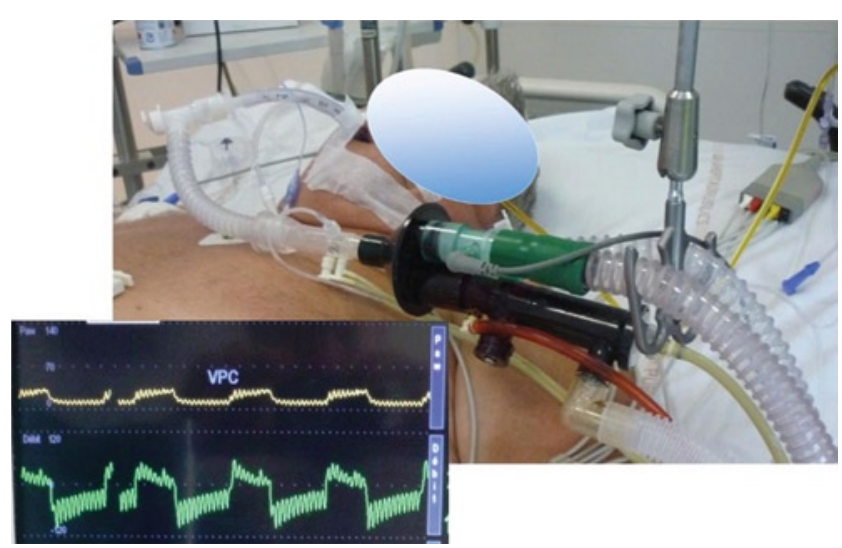

Fig. 3. Ventilation à percussions intrapulmonaires (IPV) couplée à la ventilation conventionnelle

Un cône à trois entrées permet de superposer l'IPV à la ventilation conventionnelle. Noter l'apparition des percussions sur l'écran du respirateur.

parois bronchiques (Fig. 4). Ce mécanisme favorise la mobilisation des sécrétions bronchiques et donc le recrutement de territoires pulmonaires obstrués [13]. Ces percussions génèrent une pression expiratoire positive (PEP) dans les voies aériennes [14,15], au minimum de 2 à $3 \mathrm{cmH}_{2} \mathrm{O}$ [3].

Durant une séance de drainage bronchique, l'IPV peut transitoirement assurer un support ventilatoire efficace chez des patients habituellement dépendant d'une ventilation mécanique, mais stables et dont la PEP est inférieure à $7 \mathrm{cmH}_{2} \mathrm{O}$. Il est alors possible de débrancher le patient 

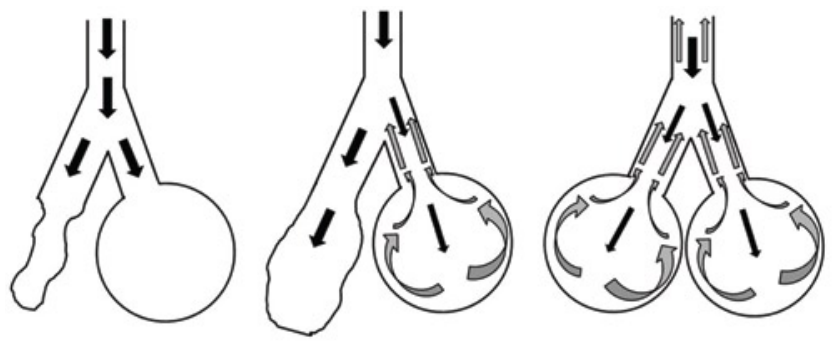

Fig. 4. Modélisation de l'action de la ventilation à percussion intrapulmonaire (IPV)

La succession des percussions crée au centre de la lumière bronchique un front de gaz qui progresse vers la périphérie du poumon, tandis qu'un contre-débit rétrograde s'établit de la périphérie vers la bouche, le long des parois bronchiques. Ce mécanisme favorise la mobilisation des sécrétions bronchiques et le recrutement de territoires pulmonaires obstrués.

du respirateur pendant la séance d'IPV (Fig. 2). Dans cette situation, il a été montré que $\mathrm{PaO}_{2}$ et $\mathrm{PaCO}_{2}$ restaient stables $[5,16,17]$ et que l'hémodynamique n'était pas altérée, y compris chez des patients présentant une dysfonction cardiaque [18].

Lorsque le niveau de PEP du respirateur est supérieur à $7 \mathrm{cmH}_{2} \mathrm{O}$, il est recommandé de coupler la ventilation conventionnelle à l'IPV par l'intermédiaire d'un cône à trois entrées (Fig. 3) et si possible d'utiliser un mode ventilatoire régulé en pression (Fig. 5).

Aucun travail publié n'a étudié le lien entre les réglages de l'IPV et ses effets intrapulmonaires in vivo. Seul un travail sur poumon test a analysé les effets de différentes combinaisons des paramètres de l'IPV [19]. Ce travail a montré :

- qu'une augmentation de la fréquence des percussions engendrait une augmentation de la pointe du pic de pression et de la PEP, associées à une diminution du volume courant délivré ;

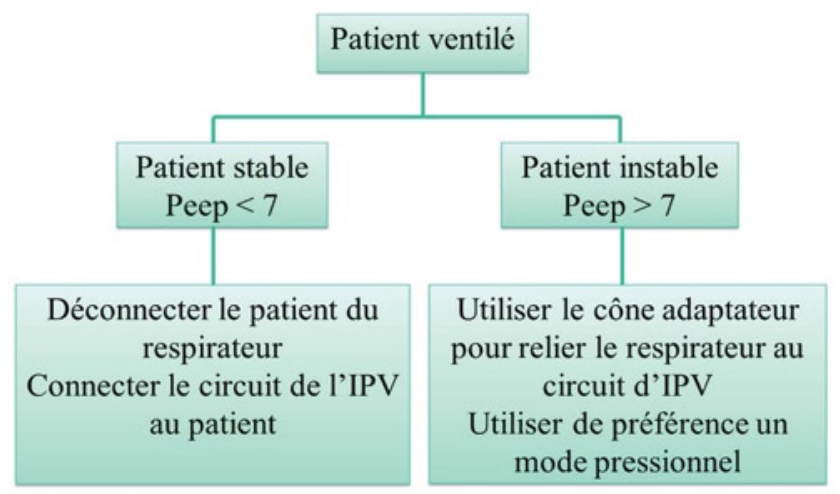

Fig. 5. Arbre décisionnel d'utilisation invasive de la ventilation à percussion intrapulmonaire (IPV)

Peep (ou PEP) : pression expiratoire positive (positive end expiratory pressure).
- qu'en revanche, une augmentation du rapport entre le temps inspiratoire et le temps expiratoire (I/E) de chaque percussion diminuait l'effet percussion, tout en augmentant la PEP. Le débit expiratoire augmentait en parallèle, ce qui théoriquement peut augmenter le déplacement du mucus. Ces modifications du rapport I/E n'ont pas eu d'impact sur le volume courant ;

- enfin, qu'une augmentation de la pression délivrée engendrait une augmentation des trois effets : PEP, volume courant et effet percussion. La PEP n'a pas dépassé $6 \mathrm{cmH}_{2} \mathrm{O}$ dans ce travail au banc, sauf avec le Pegaso ${ }^{\circledR}$ (Dima, Italia) avec lequel la PEP a pu dépasser $15 \mathrm{cmH}_{2} \mathrm{O}$.

\section{Pour : arguments en faveur de l'utilisation de l'IPV en réanimation}

L'IPV a été proposé en cas d'échec des traitements conventionnels de drainage bronchique et pour la prise en charge des exacerbations de BPCO. Une diminution de l'incidence des pneumopathies acquises sous ventilation mécanique (PAVM) et de la durée de séjour en réanimation ont été rapportées lors de l'emploi systématique de l'IPV chez des patients ventilés.

\section{Utilisation de l'IPV en réanimation en cas d'échec des traitements conventionnels de drainage bronchique}

Bien que sans doute le plus utilisé, cet emploi de l'IPV a fait essentiellement l'objet de cas cliniques, chez des patients atteints de mucoviscidose [20], de maladies neuromusculaires [21] ou en phase postopératoire de plastie sur fistule trachéo-œsophagienne [21]. L'IPV permettrait de lever des atélectasies inefficacement traitées par la kinésithérapie respiratoire conventionnelle, celle-ci étant pourtant parfois associée à des supports ventilatoires plus classiques (ventilation non invasive [VNI] ou relaxateur de pression) [21]. Le drainage des sécrétions obtenu par l'IPV est parfois décrit comme spectaculaire $[20,21]$, pouvant aboutir à l'obstruction des voies aériennes proximales par mobilisation des sécrétions bronchiques [21]. Cela souligne l'importance de s'assurer, avant d'entamer le traitement par IPV, de la possibilité d'évacuer les sécrétions ainsi mobilisées vers les voies respiratoires proximales.

Contrairement aux autres techniques de kinésithérapie respiratoire, l'IPV dispense les thérapeutes d'une action manuelle sur la cage thoracique. Afin de limiter le risque de fracture de côte, Nino et al. [22] ont ainsi utilisé l'IPV au cours d'une affection respiratoire aiguë chez un patient présentant une ostéogenèse imparfaite. L'IPV pourrait donc avoir une place dans la prise en charge de patients présentant des traumatismes thoraciques ou en phase postopératoire de chirurgie thoracique $[23,24]$. 
L'IPV offre le grand avantage de ne nécessiter aucune adaptation spécifique au type de respiration du patient (pas de synchronisation requise), ni de coopération spécifique de sa part [25]. Varekojis et al. [4] sont allés jusqu'à écrire que l'IPV permet de réaliser une séance de désencombrement en l'absence d'un spécialiste en kinésithérapie respiratoire. L'IPV pourrait donc être utilisée chez des patients encombrés présentant une altération de la conscience.

Le support ventilatoire induit par l'IPV permet son utilisation chez des patients fatigués ou présentant une capacité respiratoire fortement réduite. Toussaint et al. ont ainsi montré, dans une étude croisée randomisée ayant inclus huit patients neuromusculaires trachéotomisés, que ces patients toléraient aisément des séances ininterrompues de 30 minutes de désencombrement, et que le poids de sécrétions récoltées était supérieur lors des séances de drainage bronchique avec vs sans IPV [17], permettant d'envisager l'IPV comme le traitement de kinésithérapie à utiliser en première intention dans cette situation.

Fujita et al. [26] ont rapporté une utilisation originale de l'IPV chez un patient intubé au cours d'une pneumonie unilatérale à Legionella. En raison de la surdistension du poumon droit sain, il a réalisé une ventilation à poumon séparé : poumon droit relié à un respirateur conventionnel, poumon gauche ventilé par IPV. Cette technique a permis de drainer des quantités abondantes de mucus en provenance du poumon gauche, facilitant la guérison du patient.

\section{IPV et prise en charge des décompensations de BPCO}

Chez des patients atteints de BPCO à l'état stable, l'IPV a permis une réduction significative du travail diaphragmatique [5]. Chez des BPCO étudiées une heure après extubation, l'IPV a entrâné une amélioration du débit expiratoire et de la pression d'occlusion des voies aériennes après 0,1 seconde (P0.1) [27]. L'IPV pourrait ainsi présenter un intérêt pour prévenir certains échecs d'extubation [27]. Chez des patients $\mathrm{BPCO}$ en exacerbation avec acidose respiratoire modérée (pH entre 7,35 et 7,38), Vargas et al. [28] ont décrit une diminution de la fréquence respiratoire, une augmentation de la $\mathrm{PaO}_{2}$ et une baisse de la $\mathrm{PaCO}_{2}$ après deux séances de 30 minutes d'IPV. L'exacerbation s'est aggravée chez six patients parmi les 17 du groupe témoin contre aucun des 16 patients du groupe IPV. Cependant, le groupe témoin de cette étude n'ayant pas bénéficié de VNI, il n'est pas exclu que le support ventilatoire et la PEP induits par l'IPV aient expliqué à eux seuls les résultats obtenus, sans effet propre des percussions.

L'IPV ne doit cependant pas être considéré comme une alternative à la VNI. La diminution du travail ventilatoire induite par l'IPV est en effet inférieure à celle obtenue sous VNI [29]. Il est par contre envisageable d'associer l'IPV aux séances de VNI lors d'exacerbations de BPCO. Dans ce contexte, Antonaglia et al. [30] ont montré une plus ample réduction de la $\mathrm{PaCO}_{2}(58 \pm 5$ vs $64 \pm 5 \mathrm{mmHg})$ et une augmentation plus importante du rapport $\mathrm{PaO}_{2} / \mathrm{FIO}_{2}(274 \pm 15$ vs $218 \pm 34 \mathrm{mmHg}$ ) dans un groupe recevant des séances d'IPV, en plus de la VNI et de la kinésithérapie respiratoire, comparé à un groupe traité de façon identique mais sans IPV. Ce résultat suppose donc un rôle spécifique des percussions allant au-delà de l'effet PEP et du support ventilatoire.

\section{IPV comme technique de recrutement pulmonaire}

En comparant l'HFPV et la pression contrôlée au banc, sur un modèle de poumon à deux compartiments hétérogènes, Lucangelo et al. ont montré que l'HFPV permettait une meilleure distribution des volumes insufflés [13]. Il est possible - bien que non démontré - que l'IPV favorise, de la même manière, la ventilation de zones pulmonaires mal ventilées en les recrutant et en limitant la surdistension des territoires pulmonaires sains. Tsuruta et al. ont utilisé l'IPV dans cette intention chez dix patients obèses intubés, présentant des atélectasies postérieures de compression rebelles à la ventilation conventionnelle et chez qui la mise en procubitus était contre-indiquée [31]. En couplant l'IPV au respirateur conventionnel en continu, il a obtenu une amélioration du rapport $\mathrm{PaO}_{2} / \mathrm{FiO}_{2}$ et de la compliance dynamique avec, sur les scanners thoraciques réalisés 24 heures après le début de ce traitement, une aération des territoires postérieurs initialement collabés. Une augmentation de la PEP ou de la pression moyenne dans les voies aériennes induite par la superposition de l'IPV à la ventilation conventionnelle pourrait éventuellement expliquer les résultats obtenus. Néanmoins, Dellamonica et al. ont montré sur banc d'essai qu'au-delà de $5 \mathrm{cmH}_{2} \mathrm{O}$ de PEP sur le respirateur conventionnel, l'adjonction de l'IPV n'augmentait pas la PEP globale [32].

Par ailleurs, dans une étude prospective randomisée incluant 40 patients en postopératoire de chirurgie cardiaque, Fraipont et al. ont rapporté une diminution de la gravité des atélectasies chez des patients ayant intégré l'IPV aux séances de kinésithérapie respiratoire classique [23]. Plusieurs travaux concordent donc pour suggérer la capacité de l'IPV à recruter des territoires pulmonaires sans augmentation des pressions moyennes [21,23,31].

\section{IPV et diminution de l'incidence des pneumonies nosocomiales}

En améliorant le drainage bronchique et le recrutement de territoires pulmonaires, l'IPV pourrait avoir un intérêt dans la prévention des PAVM. Dans une étude multicentrique randomisée incluant 46 patients trachéotomisés, sevrés de la ventilation, Clini et al. ont montré une diminution significative de l'incidence des pneumonies nosocomiales dans le 
groupe recevant deux séances quotidiennes de dix minutes d'IPV en plus des séances de kinésithérapie respiratoire [33]. Le ballonnet de la trachéotomie était dégonflé lors des séances d'IPV pour faciliter l'évacuation des sécrétions mobilisées par la bouche [34]. Dalne et al. ont comparé l'incidence des PAVM chez 106 patients adultes brûlés, intubés, répartis en trois groupes, tous soumis à la kinésithérapie respiratoire [35] : le premier en ventilation conventionnelle, le deuxième en ventilation conventionnelle associée à l'IPV et le troisième ventilé en continu par HFPV. L'incidence des pneumonies fut, respectivement, de 52,3, 39,2 et 29,5\% (groupe 1 vs 2 : $p<0,001,1$ vs $3: p<0,001,2$ vs $3: p<0,05)$.

\section{Diminution de la durée d'hospitalisation}

Dans l'étude de Vargas et al., citée précédemment [28], le séjour à l'hôpital fut significativement raccourci dans le groupe avec vs sans IPV (6,8 $\pm 1,0$ vs 7,9 jours $\pm 1,3$; $p<0,05)$. Il en est de même dans le travail d'Antonaglia et al. [30] : l'adjonction de l'IPV permit de diminuer la durée de VNI en unité de soins intensifs ainsi que le nombre de jours passés dans le service. En postopératoire de chirurgie cardiaque, Fraipont et al. ont également montré une réduction de la durée d'hospitalisation dans le groupe avec vs sans IPV $(10,4 \pm 1,0$ vs $13,0 \pm 4,9$ jours ; $p=0,009)$ [23]. Enfin, dans une étude rétrospective incluant 147 enfants en postopératoire d'arthrodèse de colonne vertébrale, soumis à l'IPV avant puis après l'extubation, Lelong-Tissier et al. ont rapporté une durée maximum d'assistance ventilatoire de 24 heures [24], là où les séries classiques rapportaient entre 3 et $45 \%$ de temps de retard d'extubation.

\section{Contre : arguments en défaveur de l'utilisation de l'IPV en réanimation}

Introduite en France dans les années 1990, l'utilisation de l'IPV souffre toujours du manque de preuves scientifiques affirmant sans conteste son efficacité. Mais ceci est également le cas général des techniques de désencombrement bronchique chez le patient ventilé, dont l'intérêt reste controversé, parfois décrites comme utiles [36,37], parfois accusées de prolonger la durée de ventilation mécanique $[38,39]$.

Si les effets intrapulmonaires de l'IPV commencent à être mieux connus [19], c'est essentiellement grâce à des travaux réalisés sur un poumon test dont la compliance et la résistance imitaient un poumon sain. Les effets de l'IPV sur un poumon dont la résistance et/ou la compliance seraient altérées n'ont pas été étudiés. Mis à part l'effet PEP, les effets intrapulmonaires de l'IPV chez des patients atteints de pathologies respiratoires sont mal connus.

Dans l'état actuel de nos connaissances, la superposition de l'IPV sur la ventilation conventionnelle ne peut pas être recommandée en pratique courante. Il a, en effet, été montré que les différents respirateurs ne réagissent pas de façon identique à cette superposition, les triggers étant régulièrement perturbés [32].

Les appareils d'IPV disponibles sur le marché ne proposent pas de monitorage, en dehors d'un simple manomètre à aiguille indiquant la pression mesurée à la sortie du circuit, assimilée à la pression présente dans les voies aériennes proximales. Les débits et volumes délivrés dans les poumons ne sont pas mesurés, de même que la fréquence et le temps inspiratoire des percussions, alors même que ces paramètres influencent grandement les effets physiques obtenus dans les poumons [19]. Cette absence de monitorage est d'autant plus gênante que les principes de l'IPV sont très éloignés d'une ventilation conventionnelle. L'IPV nécessite donc une formation à son utilisation et une surveillance clinique rigoureuse.

Le risque d'assèchement des voies aériennes des patients par une humidification insuffisante a également été soulevé [40]. Dellamonica et al. ont trouvé, in vitro, un taux d'humidité des gaz injectés insuffisant lors de la superposition de l'IPV sur une ventilation conventionnelle [32]. Il convient donc d'être prudent lors de l'utilisation de l'IPV, notamment par voie invasive et pour une durée prolongée. L'augmentation des sécrétions récoltées lors des séances d'IPV [17,26,28] est toutefois peu compatible avec un assèchement du mucus.

L'utilisation de l'IPV comme générateur d'aérosol médicamenteux a été proposée. Berlinski et Willis ont montré sur banc d'essai que le dépôt d'un aérosol était comparable entre un nébuliseur pneumatique et l'IPV couplé à un respirateur conventionnel [41]. Cependant, Reychler et al. ont montré chez des volontaires sains en ventilation spontanée que le dépôt d'un aérosol d'amikacine était plus faible avec l'IPV qu'avec un nébuliseur pneumatique [42,43]. L'IPV ne peut donc actuellement être préconisée pour nébuliser des aérosols médicamenteux.

L'IPV est une technique réputée bruyante [29], ce qui est un obstacle à sa généralisation dans des unités où le bruit et le manque de sommeil sont considérés comme sources majeures de l'inconfort des patients.

En postopératoire de chirurgie pulmonaire, la possibilité que l'IPV induise des lésions des sutures bronchiques a aussi été soulevée. Bertin et al. ont réalisé des lobectomies sur des cochons et des cadavres humains, et ont ensuite étudié les répercussions de 15 minutes d'IPV réglée à pression et percussion maximales sur les cicatrices bronchopulmonaires [44]. Les auteurs n'ont pas observé de lésion sur les sutures et ont conclu qu'en l'absence de bullage spontané, l'IPV pourrait être appliquée avec sûreté en postopératoire de chirurgie pulmonaire. Aucune étude clinique n'a toutefois validé cette indication. Seul a été rapporté un 
cas clinique d'utilisation de l'IPV en postopératoire d'une plastie sur fistule trachéo-œsophagienne [21]. Gatani et al. ont également utilisé l'IPV avec succès dans le traitement de pneumothorax localisé persistant après lobectomie, mais à six mois de la chirurgie [45].

Enfin, le coût de la technique n'a pas été évalué spécifiquement. Si l'appareil est assez coûteux, les circuits sont pour l'instant stérilisables.

\section{Tentative de synthèse}

L'IPV est utilisée en routine par certaines équipes depuis une quinzaine d'années ; très peu d'effets secondaires délétères ont été rapportés : une obstruction transitoire des voies aériennes proximales par mobilisation des sécrétions bronchiques [21] et une hémoptysie isolée chez un patient atteint de mucoviscidose [46]. À ces rares exceptions près, la bonne tolérance des séances d'IPV est rapportée par tous les auteurs, y compris chez l'enfant.

À notre connaissance, le seul travail publié sur l'IPV qui ne recommande pas son utilisation comme adjuvant au drainage bronchique est celui de Ravez et al. [47]. Dans cette étude, la clairance de particules de technétium nébulisées a été mesurée chez dix patients atteints de BPCO et quatre sujets sains non fumeurs, tous soumis à 15 minutes d'IPV. Si l'IPV a bien amélioré cette clairance, l'auteur a conclu que c'était par la sollicitation du réflexe de toux, et non par l'effet propre de la technique. Néanmoins, les résultats observés chez des patients neuromusculaires sans capacité de toux efficace vont contre cette théorie [17]. L'hypothèse a été soulevée d'une augmentation de la production de sécrétions bronchiques sous l'effet d'une irritation des voies aériennes induite par l'IPV [4]. Toutefois, des patients initialement peu sécrétants n'ont pas augmenté le poids de sécrétions récoltées après les séances d'IPV [17].

L'IPV pourrait trouver une place chez des patients incapables de participer activement, du fait de la fatigue ou de problème de compréhension, à d'autres techniques de désencombrement [25]. Elle devrait donc être comparée aux différentes techniques d'assistance à la clairance bronchique. Les techniques d'expiration forcée, d'assistance à la toux par compression thoracoabdominale ou d'insufflationexsufflation mécanique ont des avantages bien connus pour le désencombrement de sécrétions situées dans l'arbre bronchique proximal de patients chez qui la toux est inefficace [48]. Mais ces techniques sont peu efficaces pour mobiliser les sécrétions périphériques [49,50], et sont inefficaces chez les patients obstructifs présentant un collapsus trachéobronchique lors des expirations forcées [51]. Dans ces différentes situations, il convient d'utiliser des expirations lentes, avantageusement associées à un système créant une PEP, limitant ainsi le collapsus expiratoire et favorisant le drainage bronchique [52,53]. Il a été suggéré que l'IPV est une bonne alternative à ces techniques de kinésithérapie respiratoire [25,46,54].

En définitive, l'argument le plus fort s'opposant à l'utilisation de l'IPV serait l'absence de connaissance de la technique, de ses principes de fonctionnement et modalités de réglages. La formation des kinésithérapeutes est ici essentielle [55]. Il faut bien sûr respecter les contre-indications à l'utilisation de l'IPV : pneumothorax non drainé et hémoptysie sévère. En cas de toux inefficace, il est indispensable de disposer d'un système d'aspiration des sécrétions qui seraient drainées de la périphérie sans pour autant pouvoir être expectorées.

\section{Conclusion}

En l'état actuel des connaissances, l'IPV ne peut être recommandée comme thérapeutique à utiliser en première intention pour le désencombrement bronchique en réanimation, et a fortiori dans d'autres indications. De nouvelles études sont nécessaires pour définir les patients qui peuvent le mieux bénéficier de cette technique. Des travaux sont également nécessaires pour élucider ses mécanismes d'action, ses effets précis sur le désencombrement et sur l'amélioration des échanges gazeux. Un monitorage adéquat des paramètres semble indispensable à développer. Les progrès de la ventilation conventionnelle, notamment ceux de la VNI, ainsi que le prix élevé des appareils délivrant la ventilation à percussion, ont certainement freiné les travaux sur cette technique. Si l'IPV ne peut se substituer à ces traitements reconnus plus efficaces [32], il est possible qu'elle puisse utilement leur être associée [56].

Conflit d'intérêt : les auteurs déclarent ne pas avoir de conflit d'intérêt.

\section{Références}

1. Velmahos GC, Chan LS, Tatevossian R, et al (1999) Highfrequency percussive ventilation improves oxygenation in patients with ARDS. Chest 116:440-6

2. Reardon CC, Christiansen D, Barnett E, Cabral HJ (2005) Intrapulmonary percussive ventilation vs incentive spirometry for children with neuromuscular disease. Arch Pediatr Adolesc Med 159:526-31

3. Natale JE, Pfeifle J, Hommick DN (1994) Comparison of intrapulmonary percussive ventilation and chest physiotherapy: a pilot study in patients with cystic fibrosis. Chest 105:1789-93

4. Varekojis SM, Douce FH, Flucke RL, et al (2003) A comparison of the therapeutic effectiveness of and preference for postural drainage and percussion, intrapulmonary percussive ventilation, and high-frequency chest wall compression in hospitalized cystic fibrosis patients. Respir Care 48:24-8

5. Nava S, Barbarito N, Piaggi G, et al (2006) Physiological response to intrapulmonary percussive ventilation in stable COPD patients. Respir Med 100:1526-33 
6. Degreef JM, Crepin JL, Deroubaix C, et al (2003) Réduction du nombre de surinfections bronchiques par la ventilation à percussion intrapulmonaire chez des patients atteints de bronchopneumopathies chroniques obstructives (BPCO) ou de bronchectasies (DDB). Rev Mal Respir S89:129

7. Dumas de La Roque E, Bertrant C, Tandonnet O, et al (2011) Nasal high frequency percussive ventilation vs nasal continuous positive airway pressure in transient tachypnea of newborn: a pilot randomized controlled trial. Pediatr Pulmonol 46:218-23

8. Bougatef A, Casteels A, Cools P, et al (2007) High frequency percussive ventilation: principle and fifteen years of experience in preterm infants with respiratory distress syndrome. J Respir Care Appl Techn 2(S1):39-50

9. Deakins K, Chatburn RL (2002) A comparison of intrapulmonary percussive ventilation and conventional chest physiotherapy for the treatment of atelectasis in the pediatric patient. Respir Care 47:1162-7

10. Yen Ha TK, Bui TD, Tran AT, et al (2007) Atelectatic children treated with intrapulmonary percussive ventilation via a face mask: clinical trial and literature overview. Pediatr Int 49:502-7

11. Ribera Cano A, Daussac E, Bonnet S, et al (2009) Ventilation non invasive par percussion intrapulmonaire (IPV) dans les bronchoalvéolites virales. Arch Pediatr 16:732-4

12. Chang HK (1984) Mechanisms of gas transport during ventilation by high-frequency oscillation. J Appl Physiol 56:553-63

13. Lucangelo U, Accardo A, Bernardi A, et al (2010) Gas distribution in a two-compartment model ventilated in high-frequency percussive and pressure-controlled modes. Intensive Care Med 36:2125-31

14. Lucangelo U, Antonaglia V, Zin WA, et al (2004) Effects of mechanical load on flow, volume and pressure delivered by high-frequency percussive ventilation. Respir Physiol Neurobiol 142:81-91

15. Dellamonica J, Louis B, Vargas F, Brochard L (2007) Bench testing of intrapulmonary percussive ventilation added to a conventional ventilator: pressures and volumes generated. Intensive Care Med 33:S137

16. Chelha R, Warmberg C, Thille A, et al (2001) Intérêt du drainage par percussion intrapulmonaire au cours du sevrage ventilatoire en réanimation. Rev Mal Respir 18:1S20

17. Toussaint M, De Win H, Steens M, Soudon P (2003) Effect of intrapulmonary percussive ventilation on mucus clearance in duchenne muscular dystrophy patients: a preliminary report. Respir Care 48:940-7

18. Nguyen ND, Borremans M, Fuhong S, et al (2003) Does intrapulmonary percussive ventilation physiotherapy have any influence on hemodynamic? Chest 124:205 S (abst)

19. Toussaint M, Guillet MC, Paternotte S, et al (2011) Intrapulmonary effects of setting parameters in portable intrapulmonary percussive ventilation devices. Respir Care (in press)

20. Dmello D, Navak RP, Matuschak GM (2010) High-frequency percussive ventilation for airway clearance in cystic fibrosis: a brief report. Lung 188:511-3

21. Birnkrant DJ, Pope JF, Lewarski J, et al (1996) Persistent pulmonary consolidation treated with intrapulmonary percussive ventilation: a preliminary report. Pediatr Pulmonol 21:246-9

22. Nino G, McNally P, Miske LJ, et al (2009) Use of intrapulmonary ventilation (IPV) in the management of pulmonary complications of an infant with osteogenesis imperfecta. Pediatr Pulmonol 44:1151-4

23. Fraipont V, Kellens I, Weber T, et al (2004) Prospective randomized controlled study of use of intrapulmonary percussive ventilation with chest physiotherapy after cardiac surgery. Crit Care 8(S1):15 (abst)
24. Lelong-Tissier MC, Chiotasso D, Boudieres G, et al (2009) Ventilation par percussion intrapulmonaire (IPV) pour la prise en charge postopératoire des scolioses de l'enfant. Arch Pediatr 16:752-4

25. Langenderfer M (1998) Alternatives to percussion and postural drainage: a review of mucus clearance therapies: percussion and postural drainage, autogenic drainage, positive expiratory pressure, flutter valve, intrapulmonary percussive ventilation, and high-frequency chest compression with the therapy vest. J Cardiopulm Rehabil 18:283-9

26. Fujita M, Tsuruta R, Oda Y, et al (2008) Severe Legionella pneumonia successfully treated by independent lung ventilation with intrapulmonary percussive ventilation. Respirology 13:475-7

27. Vargas F, Boyer A, Bui HN, et al (2009) Effect of intrapulmonary percussive ventilation on expiratory flow limitation in chronic obstructive pulmonary disease patients. J Crit Care 24:212-9

28. Vargas F, Bui HN, Boyer A, et al (2005) Intrapulmonary percussive ventilation in acute exacerbations of COPD patients with mild respiratory acidosis: a randomized controlled trial. Crit Care 9:R382-R9

29. Dimassi S, Vargas F, Lyazidi A, et al (2011) Intrapulmonary percussive ventilation superimposed on spontaneous breathing: a physiological study in patients at risk for extubation failure. Intensive Care Med 37:1269-76

30. Antonaglia V, Lucangelo U, Zin WA, et al (2006) Intrapulmonary percussive ventilation improves the outcome of patients with acute exacerbation of COPD using helmet. Crit Care Med 34:2940-5

31. Tsuruta R, Kasaoka S, Okabayashi K, Maekawa T (2006) Efficacy and safety of intrapulmonary percussive ventilation superimposed on conventional ventilation in obese patients with compression atelectasis. J Crit Care 21:328-32

32. Dellamonica J, Louis B, Lyazidi A, et al (2008) Intrapulmonary percussive ventilation superimposed on conventional ventilation: bench study of humidity and ventilator behaviour. Intensive Care Med 34:2035-43

33. Clini EM, Antoni FD, Vitacca M, et al (2006) Intrapulmonary percussive ventilation in tracheostomized patients: a randomized controlled study. Intensive Care Med 32:1994-2001

34. Allan PF, Thurlby JR, Naworol GA (2007) Measurement of pulsatile tidal volume, pressure amplitude, and gas flow during high-frequency percussive ventilation, with and without partial cuff deflation. Respir Care 52:45-9

35. Dalne E, Van Loey C, Reper P (2000) Can high frequency percussive ventilation be used to improve airway clearance in adult ventilated patients? Intensive care Med 26:S377

36. Pattanshetty RB, Gaude GS (2010) Effect of multimodality chest physiotherapy in prevention of ventilator-associated pneumonia: a randomized clinical trial. Indian J Crit Care Med 14:70-6

37. Ntoumenopoulos G, Presneill JJ, McElholum M, Cade JF (2002) Chest physiotherapy for the prevention of ventilator-associated pneumonia. Intensive Care Med 28:850-6

38. Templeton M, Palazzo MG (2007) Chest physiotherapy prolongs duration of ventilation in the critically ill ventilated for more than 48 hours. Intensive Care Med 33:1938-45

39. Patman S, Jenkins S, Stiller K (2009) Physiotherapy does not prevent, or hasten recovery from, ventilator-associated pneumonia in patients with acquired brain injury. Intensive Care Med 35:258-65

40. Allan PF, Hollingsworth MJ, Maniere GC, et al (2009) Airway humidification during high-frequency percussive ventilation. Respir Care 54:350-8

41. Berlinski A, Willis JR (2010) Albuterol delivery via intrapulmonary percussive ventilator and jet nebulizer in a pediatric ventilator model. Respi Care 55:1699-1704 
42. Reychler G, Keyeux A, Cremers C, et al (2004) Comparison of lung deposition in two types of nebulization: intrapulmonary percussive ventilation vs jet nebulization. Chest 125:502-8

43. Reychler G, Wallemacq P, Rodenstein DO, et al (2006) Comparison of lung deposition of amikacin by intrapulmonary percussive ventilation and jet nebulization by urinary monitoring. J Aerosol Med 19:199-207

44. Bertin F, Vincent F, Guerlin A, et al (2004) Risques de l'utilisation de la ventilation à percussion intrapulmonaire en postopératoire de chirurgie pulmonaire. Rev Mal Respir 21:1S53

45. Gatani T, Martucc Ni, La Rocca A, et al (2010) Management of localized pneumothoraces after pulmonary resection with intrapulmonary percussive ventilation. Ann Thorac Surg 90:1658-61

46. Homnick DN, White F, De Castro C (1995) Comparison of effects of an intrapulmonary percussive ventilator to standard aerosol and chest physiotherapy in treatment of cystic fibrosis. Pediatr Pulmonol 2:50-5

47. Ravez P, Richez M, Godart G, et al (1986) Effect of intermittent high-frequency intrapulmonary percussive breathing on mucus transport. Eur J Respir Dis 146:285-9

48. Bach JR, Ishikawa Y, Kim H (1997) Prevention of pulmonary morbidity for patients with duchenne muscular dystrophy. Chest 112:1024-28

49. Bach JR (1994) Update and perspective on noninvasive respiratory muscle aids. Part 2: the expiratory aids. Chest 105:1538-44
50. Hess DR (2001) The evidence for secretion clearance techniques. Respir Care 46:1276-93

51. Sivasothy P, Brown L, Smith IE, Shneerson JM (2001) Effect of manually assisted cough and mechanical insufflation on cough flow of normal subjects, patients with chronic obstructive pulmonary disease (COPD), and patients with respiratory muscle weakness. Thorax 56:438-44

52. Bellone A, Spagnolatti L, Massobrio M, et al (2002) Short term effect of expiration under positive pressure in patients with acute exacerbation of chronic obstructive pulmonary disease and mild acidosis requiring non-invasive positive pressure ventilation. Intensive Care Med 28:581-5

53. Inal-Ince D, Savci S, Topeli A, Arikan H (2004) Active cycle of breathing techniques in non-invasive ventilation for acute hypercapnic respiratory failure. Aust J Physiother 50:67-73

54. Newhouse PA, White F (1998) The intrapulmonary percussive ventilator and flutter device compared to chest physiotherapy in patients with cystic fibrosis. Clin Pediatr 37:427-32

55. Riffard G, Toussaint M (2012) Ventilation à percussions intrapulmonaires : fonctionnement et modalités de réglage. Rev Mal Respir (in press)

56. Vargas F, Hilbert G (2006) Intrapulmonary percussive ventilation and noninvasive positive pressure ventilation in patients with chronic obstructive pulmonary disease: "Strength through unity"? Crit Care Med 34:2940-5 